\title{
Factorization and Transverse Momentum in SIDIS at JLab
}

\author{
P.E. Bosted ${ }^{\mathrm{a}} *$
}

${ }^{a}$ Jefferson Lab, Newport News, Virginia 23606

Data for pion electroproduction from both hydrogen and deuterium targets satisfy factorization tests in the kinematic region $Q^{2}>1 \mathrm{GeV}^{2}, 0.15<x<0.45, W>2 \mathrm{GeV}$, $M_{x}>1.5 \mathrm{GeV}$, and $0.3<z<0.6$, for both spin-averaged and spin-dependent scattering. The $\pi^{+} / \pi^{-}$ratio of polarized SIDIS is found to exhibit a surprisingly large $p_{t}$ dependence.

\section{INTRODUCTION}

The process of semi-inclusive deep-inelastic lepton scattering (SIDIS) from nucleons has been shown [1] to factorize, in the high energy limit, into a product of two processes: lepton-quark scattering, followed by hadronization of the struck quark into the observed hadrons. At lower energies of the exchanged virtual photon or the observed hadron, this factorization ansatz is expected to break down, due to the effects of final state interactions, resonant nucleon excitation [2], and fragmentation of the remnant target quark, among other possibilities. Since SIDIS is a useful process to determine the both the spin-averaged and polarized flavor composition of nucleons, it is of practical importance to determine the kinematic regime where factorization works, particularly as the highest available luminosity is at Jefferson Lab, where the electron beam energy is limited to 6 $\mathrm{GeV}$ at present.

In the factorization approach, a simplified form for the SIDIS cross section is:

$d \sigma \sim \sum_{q} e_{q}^{2} q\left(x, Q^{2}\right) D_{q \rightarrow \pi}\left(z, Q^{2}\right) b e^{-b p_{T}^{2}} \frac{1+A \cos (\phi)+B \cos (2 \phi)}{2 \pi}$

where the fragmentation function $D_{q \rightarrow \pi}\left(z, Q^{2}\right)$ gives the probability for a quark to evolve into a pion detected with a fraction $z$ of the quark (or virtual photon) energy, $z=$ $E_{\pi} / \nu$. The parton distribution functions $q\left(x, Q^{2}\right)$ are the usual functions depending on the Bjorken variable $x$ and the four-momentum transfer squared $Q^{2}$. A consequence of this factorization is that the fragmentation function is independent of $x$, and the parton distribution function is independent of $z$. Both the quark distribution functions and the fragmentation functions, however, depend on $Q^{2}$ through logarithmic $Q^{2}$ evolution.

SIDIS has also been shown recently [3] to be a useful tool to investigate orbital motion of quarks, as reflected in their intrinsic transverse momenta distributions. The transverse momentum dependence of the quark distributions and fragmentation functions need not be universal, as implied in Eq. 1.

${ }^{*}$ The Southeastern Universities Research Association (SURA) operates the Thomas Jefferson National Accelerator Facility for the United States Department of Energy under contract DE-AC05-84ER40150 


\section{SPIN-AVERAGED STUDIES}

This study uses preliminary results from Jefferson Lab Hall C experiment E00-108 [4]. Incident $5.5 \mathrm{GeV}$ electrons were scattered from a 4-cm-long liquid hydrogen or deuterium target and detected in the Short Orbit Spectrometer (SOS). The central SOS momentum remained constant throughout the experiment, with a value of $1.7 \mathrm{GeV}$. The electroproduced mesons (predominantly pions) were detected in the High Momentum Spectrometer (HMS), with momenta ranging from 1.3 to $4.1 \mathrm{GeV} / \mathrm{c}$. The experiment consisted of two parts: i) at a fixed electron kinematics of $\left(x, Q^{2}, W\right)=\left(0.32,2.30 \mathrm{GeV}^{2}, 2.3 \mathrm{GeV}\right)$ the central HMS momentum was varied to cover a range of $0.3<z<1.0$ ("z-scan"); ii) at $z=0.55$, the electron scattering angle was varied, at constant momentum transfer angle, to span a range in $x$ from 0.2 to 0.58 (with corresponding $Q^{2}$ from 2.30 to $3.80 \mathrm{GeV}^{2}$ and total invariant mass $W$ from 2.5 to $2.0 \mathrm{GeV}$ )( "x-scan"). All measurements were performed for both positively-charged $\pi^{+}$and negatively-charged $\pi^{-}$. Small corrections were made for mis-identified elections and pions, contributions from the target endcaps, and random coincidences. Radiative corrections to the SIDIS process were applied using the peaking approximation as implemented in a Monte-Carlo simulation. In addition, a subtraction was made of radiated events coming from the exclusive reactions $e+p \rightarrow e^{\prime}+\pi^{+}+n$ and $e+n \rightarrow e^{\prime}+\pi^{-}+p$, using an interpolation between the low- $W^{2}$, low- $Q^{2}$ fits of MAID model [5] and the higher- $W^{2}$ data of Brauel et al. [6]. Optionally, events from diffractive $\rho$ production were subtracted using PYTHIA [7] with parameters from the HERMES collaboration $[8,9]$. A small $2 \%$ correction to the deuterium data was made to account for final-state interactions.

The ${ }^{1,2} \mathrm{H}\left(\mathrm{e}, \mathrm{e}^{\prime} \pi^{ \pm}\right) X$ cross sections as measured at $x=0.32$ are compared with the results of a simulation in Fig. 1, as a function of $z$. The simulation used the factorization ansatz with CTEQ5 NLO parton distribution [10], and the fragmentation function parameterization for $D_{q \rightarrow \pi}^{+}\left(z, Q^{2}\right)+D_{q \rightarrow \pi}^{-}\left(z, Q^{2}\right)$ from Binnewies et al. [11], where $D^{+}\left(D^{-}\right)$ are the favored (unfavored) fragmentation function. The $D^{-} / D^{+}$ratio was taken from HERMES [12], as was the $p_{t}$-dependence [8]. The general agreement between data and simulation is excellent for $z<0.6$. The missing mass of the residual system $X$ squared, $M_{x}^{2}$, is almost directly related to $z$, namely $M_{x}^{2}=M^{2}+Q^{2}(1 / x-1)(1-z)$, because $p_{T} \sim$ 0 in the chosen kinematics. Hence, the large "rise" in the data with respect to the simulation at $z>0.7(z>0.65)$ for $\pi^{+}\left(\pi^{-}\right)$mainly reflects the $N-\Delta(1232)$ region. Indeed, if one considers a ${ }^{1} \mathrm{H}\left(\mathrm{e}, \mathrm{e}^{\prime} \pi^{-}\right) X$ spectrum as function of missing mass of the residual system $X$, one sees only one prominent resonance region, the $N-\Delta$ region. Apparently, for $M_{x}>1.5 \mathrm{GeV}^{2}$, there are already sufficient resonances to render a spectrum mimicking the smooth $z$-dependence as expected from the factorization-based simulation. This can be interpreted as another manifestation of quark-hadron duality [13].

To test the factorization predictions more precisely, some simple ratios were formed of the measured cross sections, insensitive to the fragmentation process (assuming charge symmetry) at leading order in $\alpha_{s}$. If one neglects strange quarks and any $p_{T}$-dependence to the parton distribution functions, these ratios can simply be expressed in terms of $u$ and $d$ parton distributions, as follows

$$
\frac{\sigma_{p}\left(\pi^{+}\right)+\sigma_{p}\left(\pi^{-}\right)}{\sigma_{d}\left(\pi^{+}\right)+\sigma_{d}\left(\pi^{-}\right)}=\frac{4 u(x)+\bar{u}(x)+d(x)+\bar{d}(x)}{5(u(x)+d(x))}
$$




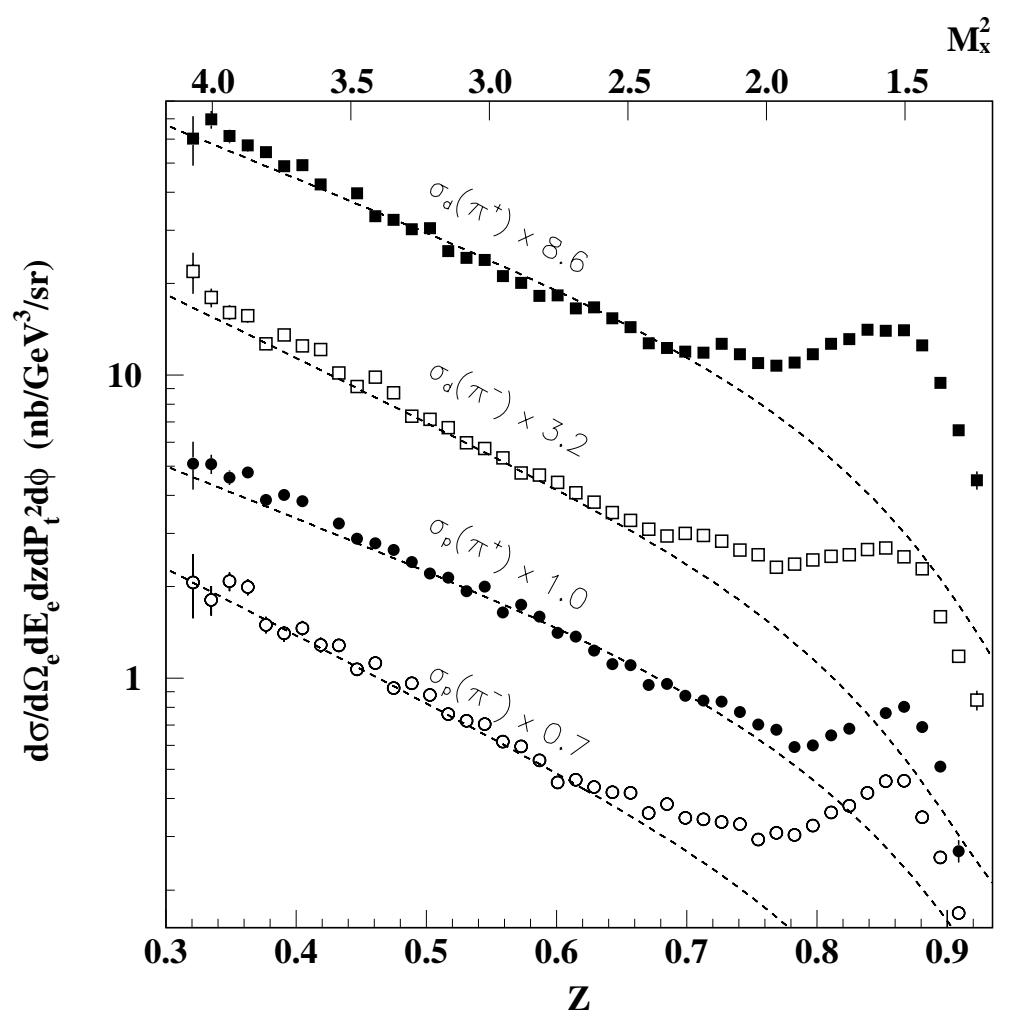

Figure 1. Preliminary ${ }^{1,2} \mathrm{H}\left(\mathrm{e}, \mathrm{e}^{\prime} \pi^{ \pm}\right) \mathrm{X}$ cross sections [4] at $x=0.32$ as a function of $z$ in comparison with simulations (dashed curves) starting from a fragmentation ansatz (see text). The various cross sections have been multiplied as indicated for clarity.

$$
\frac{\sigma_{p}\left(\pi^{+}\right)-\sigma_{p}\left(\pi^{-}\right)}{\sigma_{d}\left(\pi^{+}\right)-\sigma_{d}\left(\pi^{-}\right)}=\frac{4 u_{v}(x)-d_{v}(x)}{3\left(u_{v}(x)+d_{v}(x)\right)},
$$

with the notation $\sigma_{p}\left(\pi^{+}\right)$referring to the $\pi^{+}$pion electroproduction cross section off the proton, $u=u_{v}+\bar{u}, d=d_{v}+\bar{d}$, and the $Q^{2}$-dependence left out of these formulas for convenience. Both ratios should be independent of $z$ and agree in magnitude with the results of previous fits to the quark distribution functions (PDFs). The experimental results for these ratios are shown in Fig. 2, with the solid (open) symbols reflecting the data after (before) subtraction of the diffractive $\rho$ contributions (which pretty much cancel in the super-ratios). The data are remarkably independent of $z$, except for $z>0.7$ where the $N-\Delta$ resonance region again appears to play a dominant role. The data are also remarkably consistent in magnitude, for $z<0.7$, with the factorization-based predictions shown by the shaded bands, with the range representing the uncertainty in the PDFs.

Using the deuterium data only, the ratio of unfavored to favored fragmentation functions $D^{-} / D^{+}$can be extracted. This ratio is, to a good approximation, given by $D^{-} / D^{+}=$ $(4-r) /(4 r-1)$ where $r=\sigma_{d}\left(\pi^{+}\right) / \sigma_{d}\left(\pi^{-}\right)$. In the high-energy limit, this ratio should depend on on $z$ (and $Q^{2}$ ), but not on $x$. The results are shown as both a function of $x$ (at fixed $z$ ) and $z$ (at fixed $x$ ) in Fig. 3, with the closed (open) symbols reflecting the data 
P.E. Bosted

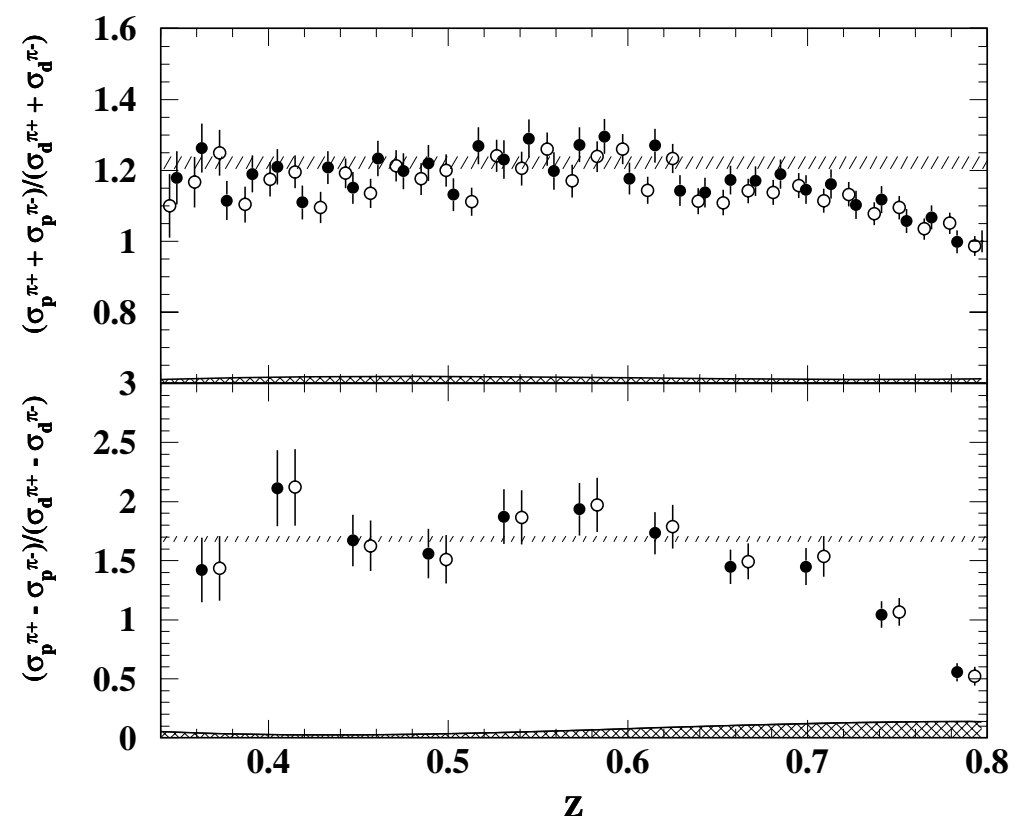

Figure 2. The ratio of proton to deuterium of preliminary results [4] for the sum (top) and difference (bottom) of $\pi^{+}$and $\pi^{-}$cross sections as a function of $z$, at $x=0.32$. Closed (open) symbols reflect data before (after) events from coherent $\rho$ production are subtracted (see text). The symbols have been sightly offset in $z$ for clarity. The shaded bands represent a range in predictions with different PDF parametrizations.

before (after) subtraction of the diffractive $\rho$ contributions. In this case the $\rho$ contribution is quite significant, and the systematic error is probably of the same order as the size of the correction itself. The present values at $z=0.55$ are indeed approximately independent of $x$ (at least for $x>0.3$ ), and are also numerically close to those obtained at HERMES [12] and EMC [14] at higher average values of $W$ and $M_{x}$, again supporting the factorization ansatz in the Jefferson Lab kinematic region. The results at $x=0.3$ are also in reasonable agreement with the HERMES data as a function of $z$, although the present results are consistently a bit larger. This is quite remarkable given that the JLab data cover the full resonance region, $0.88<M_{x}^{2}<4.2 \mathrm{GeV}^{2}$. Apparently, there is some mechanism at work, such as the SU(6) symmetric quark model [15] that removes the resonance excitations in the $\pi^{+} / \pi^{-}$ratio, and hence the $D^{-} / D^{+}$ratio. Note that all data disagree with the Field and Feynman expectation $\left.[16] D^{-} / D^{+}=(1-z) / 1+z\right)$. 

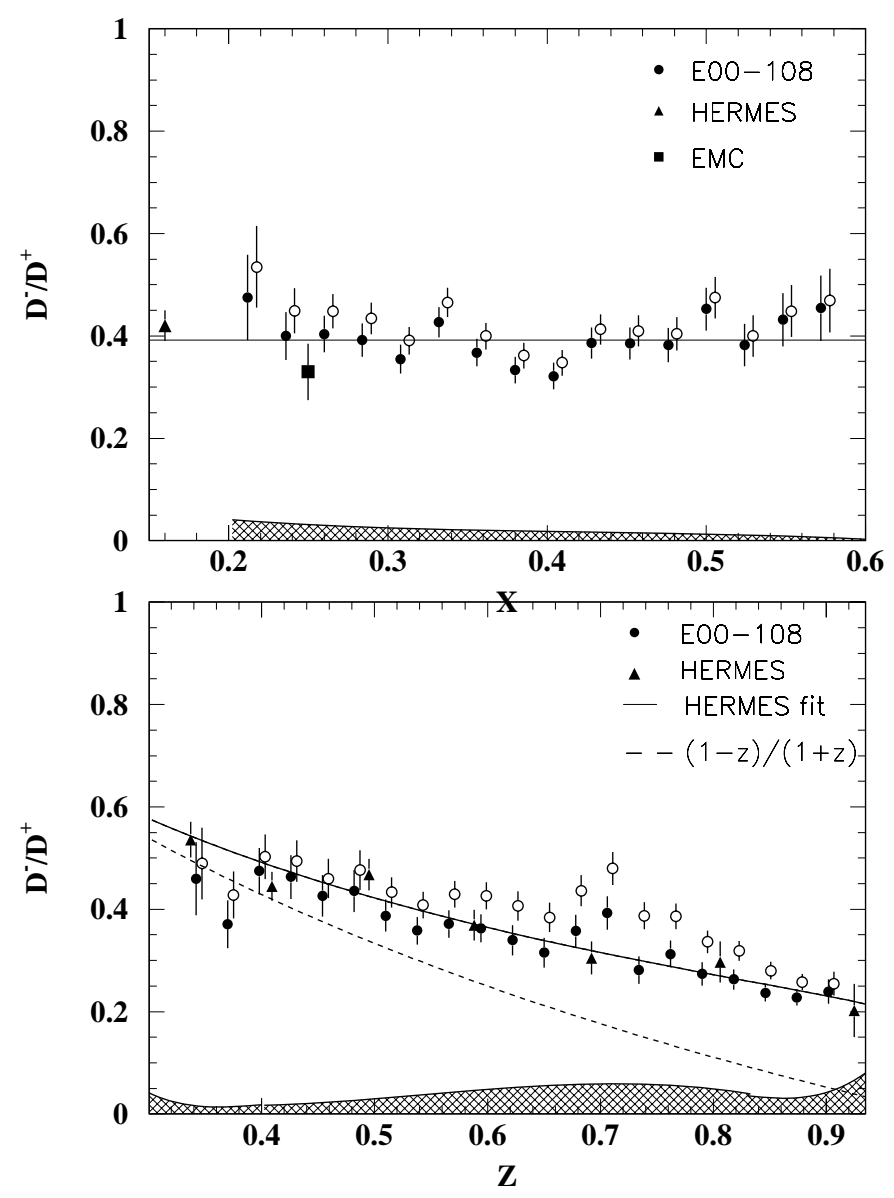

Figure 3. Top: Preliminary ratio [4] of unfavored to favored fragmentation function $D^{-} / D^{+}$as a function of $x$ at $z=0.55$, evaluated as discussed in the text. Closed (open) symbols reflect data before (after) events from coherent $\rho$ production are subtracted. Also shown are data from HERMES [12] and EMC [14]. Bottom: Same as Top, but now as a function of $z$ for $x=0.32$. The solid curve is a fit to the HERMES data, while the dashed curve represents an early QCD expectation [16].

\section{POLARIZED SIDIS}

Double-spin asymmetries in SIDIS at Jefferson Lab have recently been extracted [17]. Very briefly, in this experiment the CEBAF Large Acceptance Spectrometer [18] in Hall $\mathrm{B}$ was used to measure spin asymmetries in the scattering of longitudinally polarized electrons from longitudinally polarized protons and deuterons. The data were collected in 2001 using an incident beam energy of $5.7 \mathrm{GeV}$. Beam currents ranged from 1 to 5 $\mathrm{nA}$, and the beam polarization averaged $70 \%$. The detector package [18] allowed clean identification of electrons scattered at polar angles between 8 and 45 degrees. Charge pions were identified with a set of time-of-flight counters, and neutral pions from two photons detected in the electromagnetic calorimeter. Ammonia, polarized via Dynamic Nuclear Polarization, was used to provide polarized protons and deuterons, using the ${ }^{15} \mathrm{NH}_{3}$ and ${ }^{15} \mathrm{ND}_{3}$ isotopes, respectively. The average target polarization was about $75 \%$ 
for the proton and about $25 \%$ for the deuteron. The cuts $Q^{2}>1 \mathrm{GeV}^{2}, W>2 \mathrm{GeV}$, and $M_{x}>1.4 \mathrm{GeV}$ were applied to all data. The asymmetry dilution due to unpolarized nucleons in the target was determined from a fit to recent unpublished data on the nuclear dependence of SIDIS in CLAS (W. Brooks, spokesperson).
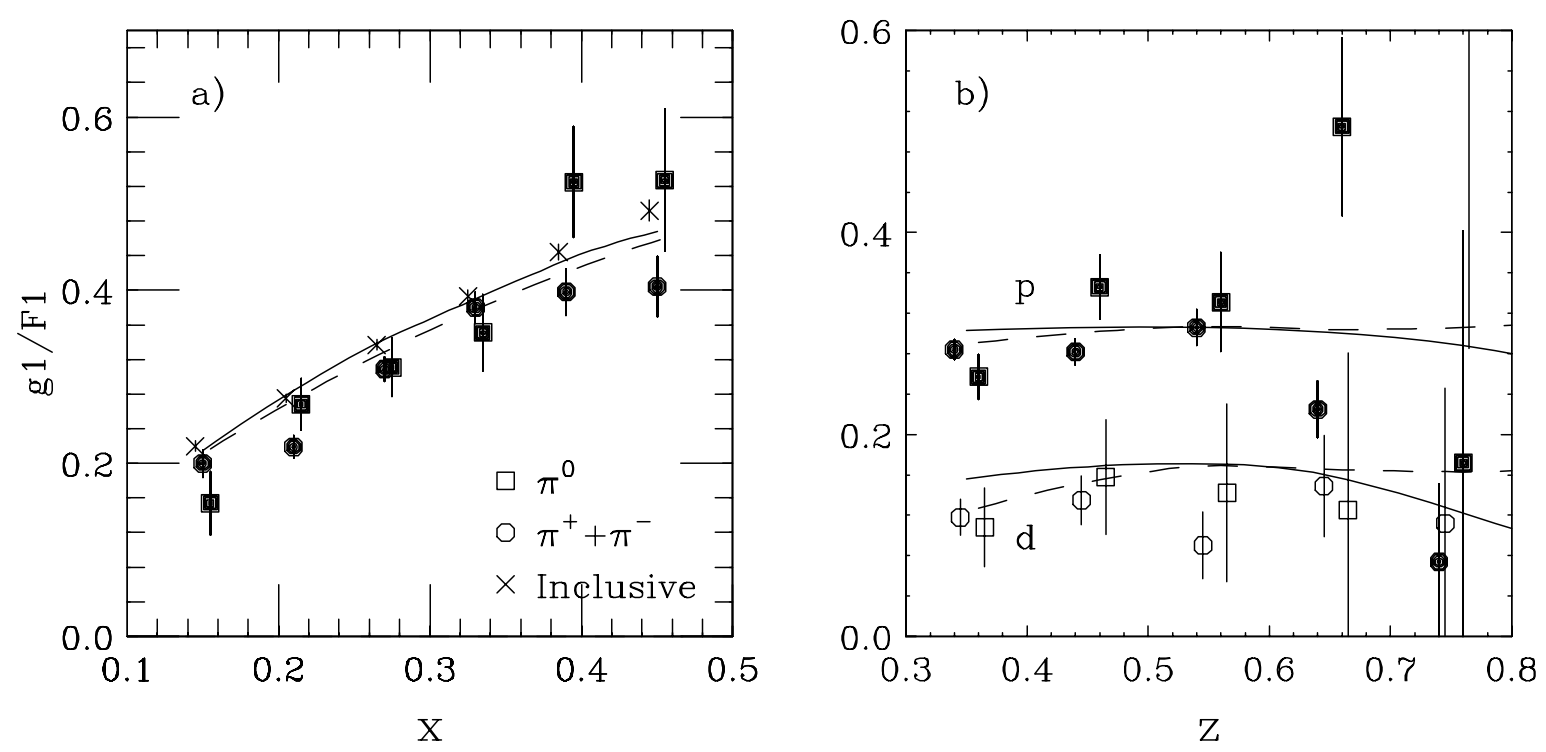

Figure 4. a) Preliminary CLAS results for proton $g_{1} / F_{1}$ inclusive (crosses) and for SIDIS $\pi^{0}$ (squares) and the sum of $\pi^{+}$and $\pi^{-}$(circles) as a function of $x$, averaged over other kinematic variables as indicated in the text. Model predictions are shown for $\pi^{0}$ (dashed lines) and the sum of $\pi^{+}$and $\pi^{-}$(solid lines). b) Same as a) but as function of $z$. Inclusive results are not shown. The open symbols and lower pair of curves are for the deuteron.

In the limit of $u$ and $d$ valence quark dominance, it can be shown that the factorization ansatz leads to the prediction that $g_{1} / F_{1}$ for inclusive scattering, for SIDIS for the sum of $\pi^{+}+\pi^{-}$, and for SIDIS for $\pi^{0}$ should be identical. The data for these three quantities, averaged over $Q^{2}>1 \mathrm{GeV}^{2}, 0.3<z<0.7, \phi$, and $P_{t}<1 \mathrm{GeV}$, are shown for the proton as a function of $x$ in Fig. 4a. We observe that the three sets of data in fact agree with each other, except that the $\pi^{+}+\pi^{-}$data are systematically a bit low. Also shown are the predictions of the Lund String Model, using polarized and unpolarized quark distributions from GRVS and GRV, and including the effects of electron radiation. The predictions are in good agreement with the data and factorization predictions for $\pi^{0}$ and inclusive, but a bit higher than the $\pi^{+}+\pi^{-}$data, roughly independent of $x$.

The data and predictions are plotted as a function of $z$ for both the proton and deuteron in Fig. 4b. We again see that the $\pi^{+}+\pi^{-}$and $\pi^{0}$ results agree with each other and the predictions, up to $z=0.6$. For $z>0.6$ or so, there appears to be a clear breakdown of the factorization ansatz, in spite of having already applied the cut $M_{x}>1.4 \mathrm{GeV}$. In particular, the $\pi^{+}+\pi^{-}$data are quite low. This may be due to the influence of diffractive 
$\rho$ production (largest at high $z$ ), or the radiative tails of exclusive single pion production (also largest at high $z$ ), as the data were not corrected for either contribution.
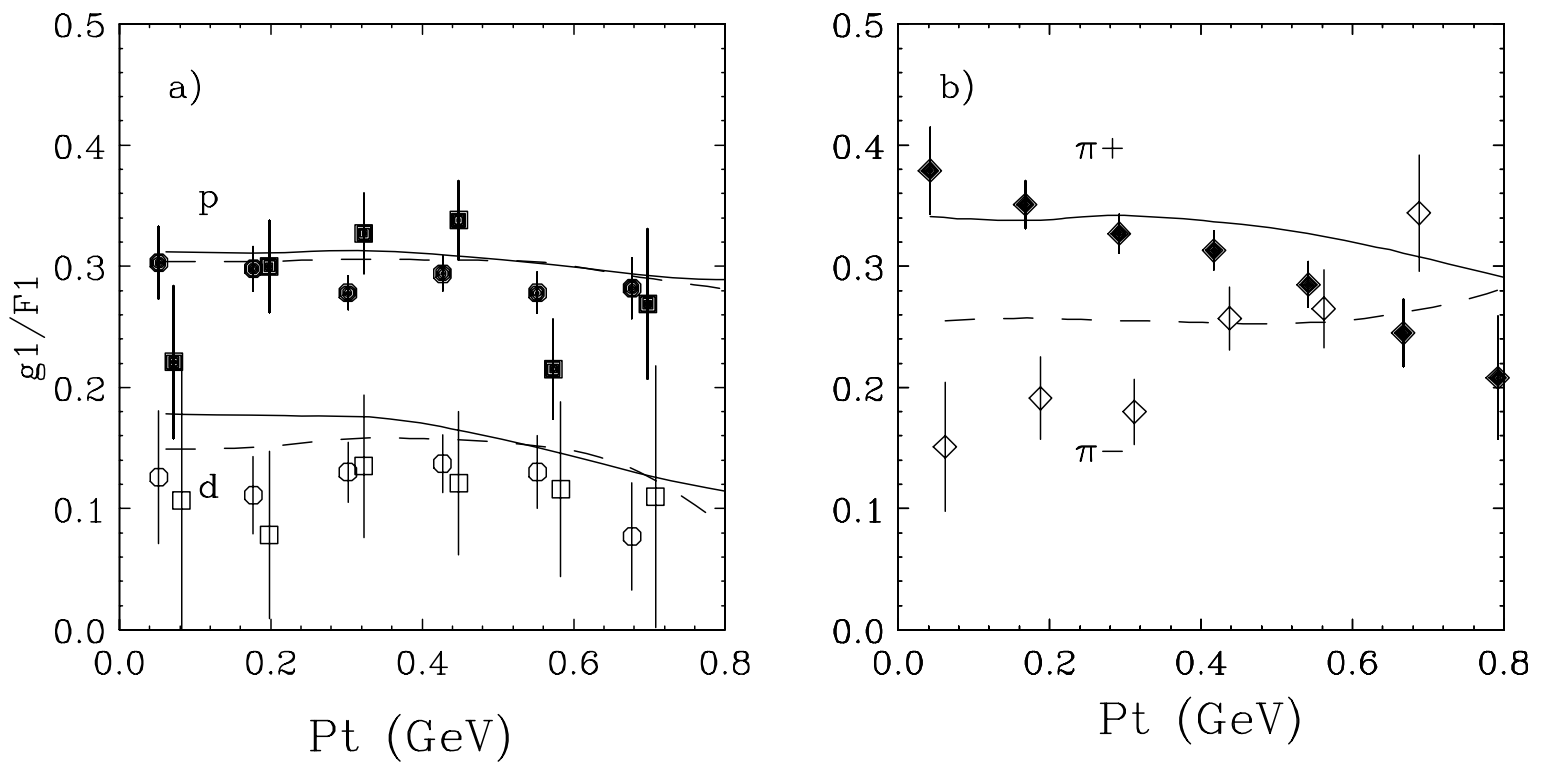

Figure 5. a) Same as Fig. 4b, but as a function of $P_{t}$. b) Proton results for SIDIS for $\pi^{+}$ (solid triangles) and $\pi^{-}$(open triangles) as a function of $P_{t}$, compared to corresponding models (solid and dashed curves, respectively).

Finally, the data and predictions are examined as a function of $P_{t}$ in Fig. 5 (with the cut $0.3<z<0.7$ ). For both targets, the $\pi^{+}+\pi^{-}$and $\pi^{0}$ results agree with each other and with the predictions pretty well and are independent of $P_{t}$, again supporting the factorization ansatz. However, the proton data for SIDIS $\pi^{+}$and $\pi^{-}$individually appear to have a significant $P_{t}$ dependence, with the trend being opposite for $\pi^{+}$and $\pi^{-}$. This is not predicted by the model to occur until $z>0.5$, and can not be easily explained by a difference in the intrinsic $<k_{t}>$ distributions of polarized and unpolarized PDFs or diffractive $\rho$ contributions (both of which typically result in the same sign $P_{t}$ slope for $\pi^{+}$and $\pi^{-}$). A more natural explanation would be a broader $P_{t}$ distribution for the unfavored fragmentation function compared to the favored one. If this were the case, it should also be seen in the $D^{-} / D^{+}$ratio extracted from unpolarized SIDIS on the deuteron, but unfortunately the Hall $\mathrm{C}$ data do not have a large enough coverage in $P_{t}$ to test this hypothesis. Another possibility are the radiative tails from exclusive pion production, which have not been corrected for in the present analysis.

\section{SUMMARY}

Tests of the factorization ansatz in SIDIS show agreement with predictions in the kinematic region $0.15<x<0.45, Q^{2}>1 \mathrm{GeV}^{2}, W>2 \mathrm{GeV}, M_{x}>1.5 \mathrm{GeV}, 0.3<z<0.6$, 
and $P_{t}<1 \mathrm{GeV}$. Tests clearly break down at higher $z$ and/or lower $M_{x}$. Surprisingly a large $P_{t}$ dependence is seen in $g_{1} / F_{1}$ for $\pi^{+}$and $\pi^{-}$individually, with opposite slopes for each. A big increase in statistical accuracy is expected for SIDIS $g_{1} / F_{1}$ in 2008 with $6 \mathrm{GeV}$ data at JLab, which will help to clarify the situation, and which will provide useful information for the flavor decomposition of the polarized PDFs. Even bigger improvements are expected once $11 \mathrm{GeV}$ beam become available.

\section{REFERENCES}

1. X. Ji, J.P. Ma, F. Yuan, Phys. Lett. B 597, 299 (2004).

2. W. Melnitchouk, AIP Conf. Proc. 588 (2001) 267.

3. S. Brodsky et al., Phys. Lett. B 530, 99 (2002); X. Ji, F. Yuan, Phys. Lett. B 543, 66 (2004); J. Collins, Phys. Lett. B 536,43 (2002).

4. T. Navasardyan et al., to be submitted to Phys. Rev. Lett. 2006.

5. D. Drechsel, S.S. Kamalov, and L. Tiator Nucl. Phys. A645 (1999) 145.

6. P. Brauel et al., Z. Physik C 3 (1979) 101.

7. T. Sjostrand, L. Lonnblad, S. Mrenna, and P. Skands, hep-ph/0308153 (2003).

8. B. Hommez, Ph.D. Dissertation, Gent University (2003), unpublished.

9. M. Tytgat, Ph.D. Dissertation, Gent University (2001), unpublished.

10. H.L. Lai et al., Eur. Phys. J. C12 (2000) 375.

11. J. Binnewies, B. A. Kniehl, and G. Kramer, Phys. Rev. D 52 (1995) 4947.

12. P. Geiger, Ph.D. Dissertation, Heidelberg University(1998), unpublished.

13. W. Melnitchouk, R. Ent, C. Keppel, Phys. Rep. 406, 127 (2005).

14. J.J. Aubert et al., Phys. Lett. B 160 (1985) 417.

15. F.E. Close and N. Isgur, Phys. Lett. B 509 (2001) 81.

16. R.D. Field and R.P. Feynman, Nucl. Phys. B136 (1978) 1.

17. P. Bosted et al., to be submitted to Phys. Rev. 2006.

18. B.A. Mecking, et al., Nucl. Instr. Meth., 503, 513 (2003). 